\title{
Gradient Flow for the Willmore Functional
}

\section{ERNST KUWERT AND REINER SCHÄTZLE}

We consider two-dimensional, compact immersed surfaces in $\mathbb{R}^{n}$ moving by the gradient of the $L^{2}$ integral of their curvature. It is not known whether solutions to this fourth-order, geometric evolution equation may develop singularities in finite time. We give a lower bound on the lifespan of a smooth solution, which depends only on how much the curvature of the initial surface is concentrated in space.

\section{Introduction.}

The Willmore energy of a closed, immersed surface $f: \Sigma \rightarrow \mathbb{R}^{n}$ with induced area measure $d \mu$ is given by

$$
\mathcal{W}(f)=\int_{\Sigma}\left|A^{0}\right|^{2} d \mu
$$

where $A^{0}=A-\frac{1}{2} g \otimes H$ is the tracefree part of the second fundamental form $A$. In his paper [14] Willmore used the $L^{2}$ integral of the mean curvature, which however differs from (1.1) only by a constant. Namely, the Gauß equations and Gauß-Bonnet imply

$$
\mathcal{W}(f)=\frac{1}{2} \int_{\Sigma}|A|^{2} d \mu-2 \pi \chi(\Sigma)=\frac{1}{2} \int_{\Sigma}|H|^{2} d \mu-4 \pi \chi(\Sigma) .
$$

The aim of this paper is to initiate a study of the $L^{2}$ gradient flow for (1.1), i.e., the geometric evolution equation

$$
\partial_{t} f=-\left(\Delta H+Q\left(A^{0}\right) H\right)=-\mathbf{W}(f) ;
$$

here the Laplacian of the normal bundle along $f$ is used and $Q\left(A^{0}\right)$ is quadratic in $A^{0}$, see (2.4). The following local existence result is standard.

Proposition 1.1. For any smooth immersion $f_{0}: \Sigma \longrightarrow \mathbb{R}^{n}$ there exists a unique, nonextendable smooth solution $f: \Sigma \times[0, T) \rightarrow \mathbb{R}^{n}$ to (1.3) with $f(\cdot, 0)=f_{0}$, where $0<T \leq \infty$. 
It is not known whether there can develop singularities under the Willmore flow in finite time. As our main result, we obtain here a lower bound on the lifespan which depends only on the concentration of curvature for the initial surface:

Theorem 1.2. Let $f_{0}: \Sigma \rightarrow \mathbb{R}^{n}$ be a smooth immersion. There are constants $\varepsilon_{0}>0, c<\infty$ depending only on $n$, such that if $\varrho>0$ is chosen with

$$
\int_{B_{\varrho}(x)}\left|A_{0}\right|^{2} d \mu_{0} \leq \varepsilon \leq \varepsilon_{0} \quad \text { for any } x \in \mathbb{R}^{n},
$$

then the maximal time $T$ of smooth existence for the Willmore flow with initial data $f_{0}$ satisfies

$$
T \geq \frac{1}{c} \varrho^{4}
$$

and one has the estimate

$$
\int_{B_{\varrho}(x)}|A|^{2} d \mu \leq c \varepsilon \quad \text { for } 0 \leq t \leq \frac{1}{c} \varrho^{4} .
$$

In the statement of the theorem the integrals should be interpreted as integrals over the preimage of $B_{\varrho}(x)$ under $f_{0}$ and $f$, respectively. Let $\varrho(t)>0$ be the biggest radius such that (1.4) holds at time $t \geq 0$ with $\varepsilon=\varepsilon_{0}$. Assuming that the flow becomes singular at $T<\infty$, it follows that $\varrho(t) \leq \sqrt[4]{c(T-t)}$ and hence at least a quantum $\varepsilon_{0}>0$ of the curvature concentrates in space.

The direct method of minimizing the Willmore energy among surfaces of fixed genus $p$ was carried out by Simon [10]. For $p=0$ and $n=3$ the critical points are all known and form an infinite sequence as shown by Bryant [1], but of course only the round spheres, i.e., $A^{0} \equiv 0$, are minimizing. Simon's work proves existence of a minimizer for any $p \geq 1$, where for $p \geq 2$ one needs an additional argument due to Kusner [4]. For an overview on Willmore surfaces we refer to Chapter 7 in [13].

Geometric evolution equations of fourth order have been considered only relatively recently. For the Willmore flow Simonett shows long term existence for initial data which are close in $C^{2, \alpha}$ to a sphere; furthermore the solution gets spherical as $t \longrightarrow \infty$ [11]. The proof uses arguments from center manifolds and follows previous work of Escher, Mayer and Simonett on the flow $\partial_{t} f=-\Delta H$ called surface diffusion [2]. 
Methods somewhat closer to ours are used by Polden in $[8,9]$ to prove long term existence and subconvergence up to translations for planar curves moving by the gradient of the functional

$$
E_{\lambda}(c)=\int_{c}\left(\varkappa^{2}+\lambda^{2}\right) d s \quad(\lambda>0) .
$$

In a forthcoming joint paper the authors have extended the results of Polden to space curves, treating also the case $\lambda=0$ with or without a length constraint [5].

For surfaces the squared curvature integral is scale invariant and in fact invariant under the full Möbius group of $\mathbb{R}^{n}$. This suggests comparing the Willmore flow to the heat flow for harmonic maps from $\mathbb{R}^{2}$ or $S^{2}$ into a Riemannian manifold, as considered for example by Struwe [12]. To a certain extent the concentration-compactness alternative proved in Theorem 1.2 affirms the analogy. Note however that there are also some differences between the Willmore flow and the harmonic map heat flow, besides the fact that the first is a fourth order equation so that tools related to the maximum principle are not available. Namely, equation (1.3) is not strictly parabolic and we are forced to pass to the evolution equation of the curvature. Furthermore, our operator is only quasilinear rather than semilinear; geometrically this means that the intrinsic geometry changes and possibly gets bad under the evolution. In the case of surfaces it becomes crucial to use only inequalities with universal constants, most importantly the Sobolev inequality of Michael and Simon [7] and interpolation inequalities as in [3]. Finally, in our case the scaling and localization is in the target of the map rather than in the domain. The last fact is a major difficulty if one tries to replace (1.4) by a local assumption.

\section{Evolution of the curvature.}

Here we derive equations for the evolution of the curvature and its derivatives, after collecting some general formulae holding for any immersion $f: \Sigma \rightarrow \mathbb{R}^{n}$. We have tried to arrange the statements in a way which facilitates possible future applications to related problems.

The basic geometric data associated to $f$ are on the one hand the induced metric $g(X, Y)=\langle D f \cdot X, D f \cdot Y\rangle$ with corresponding Levi-Civita connection $\nabla$, sectional curvature $K$ and curvature tensor $R(X, Y) Z=$ $K(g(Y, Z) X-g(X, Z) Y)$, and on the other hand the second fundamental form $A(X, Y)=D_{X, Y}^{2} f=D_{X}\left(D_{Y} f\right)-D f \cdot \nabla_{X} Y$ with mean curvature vector given by the trace $H=A\left(e_{i}, e_{i}\right)$ and trace free part $A^{0}(X, Y)=$ 
$A(X, Y)-\frac{1}{2} g(X, Y) H$. As a third object we have the normal connection $\nabla_{X} \phi=\left(D_{X} \phi\right)^{\perp}$ which acts on normal vector fields $\phi$ along $f$ and has curvature defined by $R^{\perp}(X, Y) \phi=\nabla_{X, Y}^{2} \phi-\nabla_{Y, X}^{2} \phi$ where $\nabla_{X, Y}^{2} \phi=$ . $\nabla_{X}\left(\nabla_{Y} \phi\right)-\nabla_{\nabla_{X} Y} \phi$. In the following $\left\{e_{1}, e_{2}\right\}$ always is a (locally defined) $g$ orthonormal basis on $\Sigma$ and summation over repeated indices is used. When computing tensor identities we freely use vector fields with first derivative vanishing at a given point. The quantities defined above are related by the equations of Mainardi-Codazzi, Gauß and Ricci:

$$
\begin{aligned}
\left(\nabla_{X} A\right)(Y, Z) & =\left(\nabla_{Y} A\right)(X, Z), \quad \nabla H=-\nabla^{*} A=-2 \nabla^{*} A^{0} \\
K & =\frac{1}{2}\left(|H|^{2}-|A|^{2}\right)=\frac{1}{4}|H|^{2}-\frac{1}{2}\left|A^{0}\right|^{2} \\
R^{\perp}(X, Y) \phi & =A\left(e_{i}, X\right)\left\langle A\left(e_{i}, Y\right), \phi\right\rangle-A\left(e_{i}, Y\right)\left\langle A\left(e_{i}, X\right), \phi\right\rangle \\
& =A^{0}\left(e_{i}, X\right)\left\langle A^{0}\left(e_{i}, Y\right), \phi\right\rangle-A^{0}\left(e_{i}, Y\right)\left\langle A^{0}\left(e_{i}, X\right), \phi\right\rangle .
\end{aligned}
$$

Here $\nabla^{*}$ denotes the formal adjoint of the operator $\nabla$, i.e., $\nabla^{*} \psi=$ $-\left(\nabla_{e_{i}} \psi\right)\left(e_{i}, \ldots\right)$ for any normal $l$-form along $f$. Note $\left\langle R^{\perp}(X, Y) \phi, \phi\right\rangle=0$ and in particular $R^{\perp} \equiv 0$ for $n=3$. We further define the normal endomorphism

$$
Q\left(A^{0}\right) \phi=A^{0}\left(e_{i}, e_{j}\right)\left\langle A^{0}\left(e_{i}, e_{j}\right), \phi\right\rangle
$$

and observe $0 \leq\left\langle Q\left(A^{0}\right) \phi, \phi\right\rangle \leq\left|Q\left(A^{0}\right)\right| \leq \operatorname{tr} Q\left(A^{0}\right)=\left|A^{0}\right|^{2}$, where $|\phi|=1$. An easy computation yields

$$
A^{0}\left(e_{i}, X\right)\left\langle A^{0}\left(e_{i}, Y\right), \phi\right\rangle+A^{0}\left(e_{i}, Y\right)\left\langle A^{0}\left(e_{i}, X\right), \phi\right\rangle=g(X, Y) Q\left(A^{0}\right) \phi,
$$

$$
\begin{aligned}
& A\left(e_{i}, X\right)\left\langle A\left(e_{i}, Y\right), \phi\right\rangle+A\left(e_{i}, Y\right)\left\langle A\left(e_{i}, X\right), \phi\right\rangle \\
& =g(X, Y)\left(Q\left(A^{0}\right) \phi+\frac{1}{2} H\langle H, \phi\rangle\right)+H\left\langle A^{0}(X, Y), \phi\right\rangle+A^{0}(X, Y)\langle H, \phi\rangle .
\end{aligned}
$$

Upon differentiating the identity $\left\langle\phi, D_{e_{i}} f\right\rangle=0$ one gets

$$
D_{X} \phi=\nabla_{X} \phi-\left\langle\phi, A\left(X, e_{i}\right)\right\rangle D_{e_{i}} f .
$$

We shall need to interchange the derivatives of multilinear forms on $\Sigma$ having normal values along $f$. If $\phi, \psi$ are forms of this type, we denote by $\phi * \psi$ any normal-valued, multilinear form depending on $\phi$ and $\psi$ in a universal, bilinear way. In particular we have the properties $|\phi * \psi| \leq c|\phi||\psi|$ and 
$\nabla(\phi * \psi)=\nabla \phi * \psi+\phi * \nabla \psi$. On an $l$-linear form $\phi$ the curvature $R^{l}(X, Y) \phi=$ $\nabla_{X, Y}^{2} \phi-\nabla_{Y, X}^{2} \phi$ satisfies

$$
\begin{aligned}
& \left(R^{l}(X, Y) \phi\right)\left(X_{1}, \ldots, X_{l}\right) \\
& =R^{\perp}(X, Y) \phi\left(X_{1}, \ldots, X_{l}\right)-\sum_{k=1}^{l} \phi\left(X_{1}, \ldots, R(X, Y) X_{k}, \ldots, X_{l}\right) .
\end{aligned}
$$

Using (2.2) and (2.3) we deduce

$$
R^{l}(X, Y) \phi=A * A * \phi .
$$

For this paper the precise algebraic nature of the nonlinearities in the evolution of curvature will be irrelevant, so that the $*$-notation suffices. However we will state some equations more precisely for future reference.

Lemma 2.1. For any l-linear, normal form $\phi$ we have

$$
\left(\nabla \nabla^{*}-\nabla^{*} \nabla\right) \phi=A * A * \phi-\nabla^{*} T,
$$

where

$$
T\left(X_{0}, X_{1}, \ldots, X_{l}\right)=\left(\nabla_{X_{0}} \phi\right)\left(X_{1}, X_{2}, \ldots, X_{l}\right)-\left(\nabla_{X_{1}} \phi\right)\left(X_{0}, X_{2}, \ldots, X_{l}\right) .
$$

Proof. We compute using adapted vector fields

$$
\begin{aligned}
& \left(\left(\nabla \nabla^{*}-\nabla^{*} \nabla\right) \phi\right)\left(X_{1}, \ldots, X_{l}\right) \\
& =\nabla_{e_{i}}\left(\left(\nabla_{e_{i}} \phi\right)\left(X_{1}, \ldots, X_{l}\right)\right)-\nabla_{X_{1}}\left(\left(\nabla_{e_{i}} \phi\right)\left(e_{i}, X_{2}, \ldots, X_{l}\right)\right) \\
& =\left(\nabla_{e_{i}, e_{i}}^{2} \phi\right)\left(X_{1}, \ldots, X_{l}\right)-\left(\nabla_{e_{i}, X_{1}}^{2} \phi\right)\left(e_{i}, X_{2}, \ldots, X_{l}\right) \\
& \quad+\left(\nabla_{e_{i}, X_{1}}^{2} \phi\right)\left(e_{i}, X_{2}, \ldots, X_{l}\right)-\left(\nabla_{X_{1}, e_{i}}^{2} \phi\right)\left(e_{i}, X_{2}, \ldots, X_{l}\right) \\
& =-\left(\nabla^{*} T\right)\left(X_{1}, \ldots, X_{l}\right)+\left(R^{l}\left(e_{i}, X_{1}\right) \phi\right)\left(e_{i}, X_{2}, \ldots, X_{l}\right) .
\end{aligned}
$$

The claim follows from (2.8).

We shall need two special cases of (2.9). First if we take $\phi=A$, then we have $T=0$ due to (2.1) and obtain (a rough version of) Simons' identity

$$
\Delta A=\nabla^{2} H+A * A * A \quad\left(\Delta=-\nabla^{*} \nabla\right) .
$$

Second we replace $\phi$ in (2.9) by $\nabla \phi$, where $\phi$ is a $(l-1)$-linear, normal form. We then have $T\left(X_{0}, X_{1}, X_{2}, \ldots, X_{l}\right)=\left(R^{l-1}\left(X_{0}, X_{1}\right) \phi\right)\left(X_{2}, \ldots, X_{l}\right)$, which means $T=A * A * \phi$ by (2.8) and therefore

$$
\left(\nabla \nabla^{*}-\nabla^{*} \nabla\right) \nabla \phi=A * A * \nabla \phi+A * \nabla A * \phi .
$$


In the following lemma we collect without proof the variational formulae for various geometric objects.

Lemma 2.2. Let $f: \Sigma \times I \rightarrow \mathbb{R}^{n}, I=\left(t_{1}, t_{2}\right)$, be a smooth variation with normal velocity field $V=\partial_{t} f$. Then the following equations hold (where $X, Y, Z$ do not depend on $t)$ :

$$
\begin{aligned}
\partial_{t} P= & -\partial_{t} P^{\perp}=D f \cdot(\nabla V)^{*}+\nabla V \cdot(D f)^{*}, \text { where } P, P^{\perp} \\
& \text { are the projections onto tangent space } \\
& \text { and normal space; } \\
P\left(\partial_{t} \phi\right)= & D f(\nabla V)^{*} \phi \text { for any normal } \phi \text { along } f ; \\
\partial_{t}^{\perp} \nabla_{X} \phi= & \nabla_{X} \partial_{t}^{\perp} \phi+A\left(X, e_{i}\right)\left\langle\nabla_{e_{i}} V, \phi\right\rangle+\nabla_{e_{i}} V\left\langle A\left(X, e_{i}\right), \phi\right\rangle, \\
& \text { where } \partial_{t}^{\perp}=P^{\perp} \partial_{t} \text { and } \phi \text { is normal along } f ; \\
\left(\partial_{t} g\right)(X, Y)= & -2\langle A(X, Y), V\rangle ; \\
\partial_{t}(d \mu)= & -\langle H, V\rangle d \mu ; \\
\partial_{t}\left(\nabla_{X} Y\right)= & -\left\langle\left(\nabla_{e_{i}} A\right)(X, Y), V\right\rangle e_{i}+\left\langle A(X, Y), \nabla_{e_{i}} V\right\rangle e_{i} \\
& -\left\langle A\left(X, e_{i}\right), \nabla_{Y} V\right\rangle e_{i}-\left\langle A\left(Y, e_{i}\right), \nabla_{X} V\right\rangle e_{i} ; \\
\partial_{t}^{\perp} A(X, Y)= & \nabla_{X, Y}^{2} V-A\left(e_{i}, X\right)\left\langle A\left(e_{i}, Y\right), V\right\rangle ; \\
\partial_{t}^{\perp} H= & \Delta V+Q\left(A^{0}\right) V+\frac{1}{2} H\langle H, V\rangle .
\end{aligned}
$$

The following Lemma will be needed for computing the evolution of derivatives of the curvature.

Lemma 2.3. Let $\phi$ be an $(l-1)$-form with normal values along a variation $f: \Sigma \times I \longrightarrow \mathbb{R}^{n}$ with normal velocity $\partial_{t} f=V$. If $\partial_{t}^{\perp} \phi+\Delta^{2} \phi=Y$, then $\psi=\nabla \phi$ satisfies an equation

$$
\begin{aligned}
\partial_{t}^{\perp} \psi+\Delta^{2} \psi= & \nabla Y+\sum_{i+j+k=3} \nabla^{i} A * \nabla^{j} A * \nabla^{k} \phi \\
& +A * \nabla V * \phi+\nabla A * V * \phi .
\end{aligned}
$$

Proof. Let $X_{1}, \ldots, X_{l}$ be independent of $t$ and such that $\nabla X_{k}=0$ at a given 
point and a given time. We then have

$$
\begin{aligned}
& \left(\partial_{t}^{\perp} \psi\right)\left(X_{1}, \ldots, X_{l}\right) \\
& =\partial_{t}^{\perp}\left(\left(\nabla_{X_{1}} \phi\right)\left(X_{2}, \ldots, X_{l}\right)\right) \\
& =\partial_{t}^{\perp} \nabla_{X_{1}} \phi\left(X_{2}, \ldots, X_{l}\right)-\partial_{t}^{\perp} \sum_{k=2}^{l} \phi\left(X_{2}, \ldots, \nabla_{X_{1}} X_{k}, \ldots, X_{l}\right) .
\end{aligned}
$$

In the first term we use (2.14) to obtain

$$
\begin{aligned}
& \partial_{t}^{\perp} \nabla_{X_{1}} \phi\left(X_{2}, \ldots, X_{l}\right) \\
& =\nabla_{X_{1}} \partial_{t}^{\perp} \phi\left(X_{2}, \ldots, X_{l}\right)+(A * \nabla V * \phi)\left(X_{1}, \ldots, X_{l}\right) \\
& =\left(\nabla\left(\partial_{t}^{\perp} \phi\right)\right)\left(X_{1}, \ldots, X_{l}\right)+(A * \nabla V * \phi)\left(X_{1}, \ldots, X_{l}\right) .
\end{aligned}
$$

By (2.17) the second term above has the form $A * \nabla V * \phi+\nabla A * V * \phi$. We thus arrive at

$$
\partial_{t}^{\perp} \psi+\Delta^{2} \psi-\nabla Y=\Delta^{2}(\nabla \phi)-\nabla\left(\Delta^{2} \phi\right)+A * \nabla V * \phi+\nabla A * V * \phi .
$$

Now we use (2.11) to obtain

$$
\begin{aligned}
\Delta^{2}(\nabla \phi)-\nabla\left(\Delta^{2} \phi\right)= & \Delta(\Delta(\nabla \phi)-\nabla(\Delta \phi))+\Delta(\nabla(\Delta \phi))-\nabla(\Delta(\Delta \phi)) \\
= & \Delta(A * A * \nabla \phi+A * \nabla A * \phi) \\
& +A * A * \nabla(\Delta \phi)+A * \nabla A * \Delta \phi \\
= & \sum_{i+j+k=3} \nabla^{i} A * \nabla^{j} A * \nabla^{k} \phi,
\end{aligned}
$$

which proves (2.20).

Using (1.2), (2.16) and (2.19) the first variation formula for the Willmore integral in a normal direction $\phi$ becomes

$$
\begin{aligned}
\left.\frac{d}{d \varepsilon} \mathcal{W}(f+\varepsilon \phi)\right|_{\varepsilon=0} & =\left.\frac{d}{d \varepsilon} \int_{\Sigma} \frac{1}{2}|H|^{2} d \mu\right|_{\varepsilon=0} \\
& =\int_{\Sigma}\left\langle H, \Delta \phi+Q\left(A^{0}\right) \phi\right\rangle d \mu \\
& =\int_{\Sigma}\left\langle\Delta H+Q\left(A^{0}\right) H, \phi\right\rangle d \mu
\end{aligned}
$$


which proves $\operatorname{grad}_{L^{2}} \mathcal{W}(f)=\Delta H+Q\left(A^{0}\right) H=\mathbf{W}(f)$. In the following we use the notation $P_{r}^{m}(A)$ for any term of the type

$$
P_{r}^{m}(A)=\sum_{i_{1}+\ldots+i_{r}=m} \nabla^{i_{1}} A * \nabla^{i_{2}} A * \ldots * \nabla^{i_{r}} A .
$$

Proposition 2.4. Under the Willmore flow $\partial_{t} f=-\mathbf{W}(f)$, we have the evolution equations

$$
\partial_{t}^{\perp}\left(\nabla^{m} A\right)+\Delta^{2}\left(\nabla^{m} A\right)=P_{3}^{m+2}(A)+P_{5}^{m}(A)
$$

for any $m \in \mathbb{N}_{0}$.

Proof. We proceed by induction on $m$, starting with $m=0$. Using (2.11) we have

$$
\begin{aligned}
& \Delta\left(\nabla^{2} H\right)-\nabla^{2}(\Delta H) \\
& =\left(\nabla \nabla^{*}-\nabla^{*} \nabla\right) \nabla(\nabla H)+\nabla\left(\nabla \nabla^{*}-\nabla^{*} \nabla\right) \nabla H \\
& =A * A * \nabla^{2} H+A * \nabla A * \nabla H+\nabla(A * A * \nabla H+A * \nabla A * H) .
\end{aligned}
$$

Therefore (2.18) yields

$$
\begin{aligned}
\partial_{t}^{\perp} A & =\nabla^{2}(\mathbf{W}(f))+A * A * \mathbf{W}(f) \\
& =\nabla^{2}(\Delta H)+P_{3}^{2}(A)+P_{5}^{0}(A) \\
& =\Delta\left(\nabla^{2} H\right)+P_{3}^{2}(A)+P_{5}^{0}(A) \\
& =\Delta^{2} A+P_{3}^{2}(A)+P_{5}^{0}(A),
\end{aligned}
$$

where (2.10) was used in the last step. Now let $m \geq 1$ and conclude from (2.20) using induction

$$
\begin{aligned}
& \partial_{t}^{\perp}\left(\nabla^{m} A\right)+\Delta^{2}\left(\nabla^{m} A\right) \\
& =\nabla\left(P_{3}^{m+1}(A)+P_{5}^{m-1}(A)\right)+\sum_{i+j+k=3} \nabla^{i} A * \nabla^{j} A * \nabla^{k}\left(\nabla^{m-1} A\right) \\
& \quad+A * \nabla(\mathbf{W}(f)) * \nabla^{m-1} A+\nabla A * \mathbf{W}(f) * \nabla^{m-1} A .
\end{aligned}
$$

Inserting $\mathbf{W}(f)=\Delta H+A * A * A$ yields the result. 


\section{Energy type inequalities.}

In this section we convert the evolution equations obtained in Proposition 2.4 into energy type inequalities. We start with the following general identity.

Lemma 3.1. Let $f: \Sigma \times I \rightarrow \mathbb{R}^{n}$ be a variation with $\partial_{t} f=V$ normal, and let $\phi$ be a l-linear, normal form along $f$ which satisfies $\partial_{t}^{\perp} \phi+\Delta^{2} \phi=Y$. Then for any $\eta \in C^{2}(\Sigma \times I)$ we have

$$
\begin{aligned}
& \frac{d}{d t} \int_{\Sigma} \frac{1}{2}|\phi|^{2} \eta d \mu+\int_{\Sigma}\langle\Delta \phi, \Delta(\eta \phi)\rangle d \mu-\int_{\Sigma}\langle Y, \eta \phi\rangle d \mu \\
& =\int_{\Sigma} \eta \sum_{k=1}^{l}\left\langle A\left(e_{i_{k}}, e_{j}\right), V\right\rangle\left\langle\phi\left(e_{i_{1}}, \ldots, e_{i_{k}}, \ldots, e_{i_{l}}\right), \phi\left(e_{i_{1}}, \ldots, e_{j}, \ldots, e_{i_{l}}\right)\right\rangle d \mu \\
& \quad-\int_{\Sigma} \frac{1}{2}|\phi|^{2}\langle H, V\rangle \eta d \mu+\int_{\Sigma} \frac{1}{2}|\phi|^{2} \partial_{t} \eta d \mu .
\end{aligned}
$$

Here $g\left(e_{i}, e_{j}\right)=\delta_{i j}$ locally on $\Sigma \times I$ and summation over $j, i_{\nu} \in\{1,2\}$, $1 \leq \nu \leq l$, is used.

Proof. Recalling (2.15) and (2.16) we have

$$
\begin{aligned}
& \partial_{t} \int_{\Sigma} \frac{1}{2} \eta\left\langle\phi\left(e_{i_{1}}, \ldots, e_{i_{l}}\right), \phi\left(e_{i_{1}}, \ldots, e_{i_{l}}\right)\right\rangle d \mu \\
& =-\int_{\Sigma}\left\langle\Delta^{2} \phi, \eta \phi\right\rangle d \mu+\int_{\Sigma} \eta\langle Y, \phi\rangle d \mu \\
& \quad+\int_{\Sigma} \eta \sum_{k=1}^{l}\left\langle\phi\left(e_{i_{1}}, \ldots, e_{i_{k}}, \ldots, e_{i_{l}}\right), \phi\left(e_{i_{1}}, \ldots, \partial_{t} e_{i_{k}}, \ldots, e_{i_{l}}\right)\right\rangle d \mu \\
& \quad-\int_{\Sigma} \frac{1}{2} \eta\langle H, V\rangle|\phi|^{2} d \mu+\int_{\Sigma} \frac{1}{2} \partial_{t} \eta|\phi|^{2} d \mu .
\end{aligned}
$$

In the third integral we note $g\left(\partial_{t} e_{i_{k}}, e_{j}\right)+g\left(e_{i_{k}}, \partial_{t} e_{j}\right)=-\left(\partial_{t} g\right)\left(e_{i_{k}}, e_{j}\right)=$ $2\left\langle A\left(e_{i_{k}}, e_{j}\right), V\right\rangle$, where (2.15) was used in the last step. The claim follows by symmetry in $i_{k}, j$. 
Lemma 3.2. Under the assumptions of the previous lemma, let $\eta=\gamma^{s}$ with $\gamma \in C^{2}(\Sigma \times I)$ and $s \geq 4$. Then for $c=c(n, s)$ we have

$$
\begin{aligned}
& \frac{d}{d t} \int_{\Sigma}|\phi|^{2} \gamma^{s} d \mu+\int_{\Sigma}\left|\nabla^{2} \phi\right|^{2} \gamma^{s} d \mu-\int_{\Sigma} 2\langle Y, \phi\rangle \gamma^{s} d \mu \\
& \leq \int_{\Sigma}\langle A * \phi * \phi, V\rangle \gamma^{s} d \mu+\int_{\Sigma}|\phi|^{2} s \gamma^{s-1} \partial_{t} \gamma d \mu \\
& \quad+c \int_{\Sigma}|\phi|^{2} \gamma^{s-4}\left(|\nabla \gamma|^{4}+\gamma^{2}\left|\nabla^{2} \gamma\right|^{2}\right) d \mu+c \int_{\Sigma}|\phi|^{2}\left(|\nabla A|^{2}+|A|^{4}\right) \gamma^{s} d \mu
\end{aligned}
$$

Proof. We have

$$
\begin{aligned}
& \int_{\Sigma}\left|\nabla^{2} \phi\right|^{2} \gamma^{s} d \mu \\
& \leq \int_{\Sigma}\left\langle\nabla^{2} \phi, \nabla^{2}\left(\gamma^{s} \phi\right)\right\rangle d \mu+c \int_{\Sigma}\left|\nabla^{2} \phi\right| \gamma^{s-1}|\nabla \gamma||\nabla \phi| d \mu \\
& \quad+c \int_{\Sigma}\left|\nabla^{2} \phi\right| \gamma^{s-2}\left(|\nabla \gamma|^{2}+\gamma\left|\nabla^{2} \gamma\right|\right)|\phi| d \mu \\
& \leq \int_{\Sigma}\left\langle\nabla^{2} \phi, \nabla^{2}\left(\gamma^{s} \phi\right)\right\rangle d \mu+\frac{1}{4} \int_{\Sigma}\left|\nabla^{2} \phi\right|^{2} \gamma^{s} d \mu \\
& \quad+c \int_{\Sigma}|\nabla \phi|^{2} \gamma^{s-2}|\nabla \gamma|^{2} d \mu+c \int_{\Sigma}|\phi|^{2} \gamma^{s-4}\left(|\nabla \gamma|^{4}+\gamma^{2}\left|\nabla^{2} \gamma\right|^{2}\right) d \mu .
\end{aligned}
$$

In the third term we integrate by parts to get

$$
\begin{aligned}
& \int_{\Sigma}|\nabla \phi|^{2} \gamma^{s-2}|\nabla \gamma|^{2} d \mu \\
& \leq-\int_{\Sigma}\langle\phi, \Delta \phi\rangle \gamma^{s-2}|\nabla \gamma|^{2} d \mu+c \int_{\Sigma}|\phi||\nabla \phi| \gamma^{s-3}|\nabla \gamma|^{3} d \mu \\
& \quad+c \int_{\Sigma}|\phi||\nabla \phi| \gamma^{s-2}|\nabla \gamma|\left|\nabla^{2} \gamma\right| d \mu \\
& \leq \varepsilon \int_{\Sigma}\left|\nabla^{2} \phi\right|^{2} \gamma^{s} d \mu+c(\varepsilon) \int_{\Sigma}|\phi|^{2} \gamma^{s-4}|\nabla \gamma|^{4} d \mu
\end{aligned}
$$




$$
+\frac{1}{2} \int_{\Sigma}|\nabla \phi|^{2} \gamma^{s-2}|\nabla \gamma|^{2} d \mu+c \int_{\Sigma}|\phi|^{2} \gamma^{s-2}\left|\nabla^{2} \gamma\right|^{2} d \mu .
$$

Combining the two inequalities yields

$$
\begin{aligned}
& \int_{\Sigma}\left|\nabla^{2} \phi\right|^{2} \gamma^{s} d \mu+\int_{\Sigma}|\nabla \phi|^{2} \gamma^{s-2}|\nabla \gamma|^{2} d \mu \\
& \leq \frac{3}{2} \int_{\Sigma}\left\langle\nabla^{2} \phi, \nabla^{2}\left(\gamma^{s} \phi\right)\right\rangle d \mu+c \int_{\Sigma}|\phi|^{2} \gamma^{s-4}\left(|\nabla \gamma|^{4}+\gamma^{2}\left|\nabla^{2} \gamma\right|^{2}\right) d \mu .
\end{aligned}
$$

Next using (2.11) we compute

$$
\begin{aligned}
& \int_{\Sigma}\left\langle\nabla^{2} \phi, \nabla^{2}\left(\gamma^{s} \phi\right)\right\rangle d \mu \\
& =\int_{\Sigma}\left\langle\Delta \phi, \Delta\left(\gamma^{s} \phi\right)\right\rangle d \mu+\int_{\Sigma}\left\langle A * A * \nabla \phi+A * \nabla A * \phi, \nabla\left(\gamma^{s} \phi\right)\right\rangle d \mu \\
& \leq \int_{\Sigma}\left\langle\Delta \phi, \Delta\left(\gamma^{s} \phi\right)\right\rangle d \mu \\
& \quad+\int_{\Sigma}\langle A * A * \nabla \phi, \nabla \phi\rangle \gamma^{s} d \mu+\int_{\Sigma}\langle A * \nabla A * \phi, \nabla \phi\rangle \gamma^{s} d \mu \\
& \quad+c \int_{\Sigma}|A|^{2}|\phi||\nabla \phi| \gamma^{s-1}|\nabla \gamma| d \mu+c \int_{\Sigma}|A||\nabla A||\phi|^{2} \gamma^{s-1}|\nabla \gamma| d \mu .
\end{aligned}
$$

The last two integrals are estimated as follows:

$$
\begin{aligned}
& \int_{\Sigma}|A|^{2}|\phi||\nabla \phi| \gamma^{s-1}|\nabla \gamma| d \mu \\
& \leq \varepsilon \int_{\Sigma}|\nabla \phi|^{2} \gamma^{s-2}|\nabla \gamma|^{2} d \mu+c(\varepsilon) \int_{\Sigma}|\phi|^{2}|A|^{4} \gamma^{s} d \mu, \\
& \int_{\Sigma}|A||\nabla A||\phi|^{2} \gamma^{s-1}|\nabla \gamma| d \mu \\
& \leq \int_{\Sigma}|\phi|^{2} \gamma^{s-4}|\nabla \gamma|^{4} d \mu+\int_{\Sigma}|\phi|^{2}\left(|\nabla A|^{2}+|A|^{4}\right) \gamma^{s} d \mu .
\end{aligned}
$$


Using integration by parts we obtain

$$
\begin{aligned}
& \int_{\Sigma}|A|^{2}|\nabla \phi|^{2} \gamma^{s} d \mu \\
& \leq-\int_{\Sigma}|A|^{2}\langle\phi, \Delta \phi\rangle \gamma^{s} d \mu \\
& \quad+\int_{\Sigma}(A * \nabla A * \phi * \nabla \phi) \gamma^{s} d \mu+c \int_{\Sigma}|A|^{2}|\phi||\nabla \phi| \gamma^{s-1}|\nabla \gamma| d \mu \\
& \leq \varepsilon \int_{\Sigma}\left|\nabla^{2} \phi\right|^{2} \gamma^{s} d \mu+c(\varepsilon) \int_{\Sigma}|\phi|^{2}|A|^{4} \gamma^{s} d \mu \\
& \quad+\int_{\Sigma}(A * \nabla A * \phi * \nabla \phi) \gamma^{s} d \mu+\varepsilon \int_{\Sigma}|\nabla \phi|^{2} \gamma^{s-2}|\nabla \gamma|^{2} d \mu,
\end{aligned}
$$

and

$$
\begin{aligned}
& \int_{\Sigma}(A * \nabla A * \phi * \nabla \phi) \gamma^{s} d \mu \\
& \leq \frac{1}{2} \int_{\Sigma}|A|^{2}|\nabla \phi|^{2} \gamma^{s} d \mu+c \int_{\Sigma}|\phi|^{2}|\nabla A|^{2} \gamma^{s} d \mu .
\end{aligned}
$$

Combining these inequalities yields

$$
\begin{aligned}
& \int_{\Sigma}|A|^{2}|\nabla \phi|^{2} \gamma^{s} d \mu+\int_{\Sigma}(A * \nabla A * \phi * \nabla \phi) \gamma^{s} d \mu \\
& \leq \varepsilon \int_{\Sigma}\left|\nabla^{2} \phi\right|^{2} \gamma^{s} d \mu+\varepsilon \int_{\Sigma}|\nabla \phi|^{2} \gamma^{s-2}|\nabla \gamma|^{2} d \mu \\
& \quad+c(\varepsilon) \int_{\Sigma}|\phi|^{2}\left(|\nabla A|^{2}+|A|^{4}\right) \gamma^{s} d \mu .
\end{aligned}
$$

The claim follows by inserting (3.3)-(3.6) into (3.1).

In the following we assume that $\gamma=\widetilde{\gamma} \circ f$, where $0 \leq \widetilde{\gamma} \leq 1$ and $\|\widetilde{\gamma}\|_{C^{2}\left(\mathbb{R}^{n}\right)} \leq c<\infty$. This implies $\nabla \gamma=(D \widetilde{\gamma} \circ f) D f$ and $\nabla^{2} \gamma=$ $\left(D^{2} \widetilde{\gamma} \circ f\right)(D f, D f)+(D \widetilde{\gamma} \circ f) A(\cdot, \cdot)$, and therefore we have

$$
|\nabla \gamma| \leq c, \quad\left|\nabla^{2} \gamma\right| \leq c(1+|A|) .
$$


We furthermore specialize to the Willmore flow, i.e.,

$$
\begin{aligned}
V=-\mathbf{W}(f) & =-\left(\Delta H+Q\left(A^{0}\right) H\right)=P_{1}^{2}(A)+P_{3}^{0}(A), \quad \text { and } \\
\partial_{t} \gamma & =(D \widetilde{\gamma} \circ f) \cdot\left(\Delta H+P_{3}^{0}(A)\right) .
\end{aligned}
$$

Proposition 3.3. Let $f: \Sigma \times I \rightarrow \mathbb{R}^{n}$ be a smooth Willmore flow. Then for $\gamma=\widetilde{\gamma} \circ f$ satisfying (3.7), $\phi=\nabla^{m} A$ with $m \in \mathbb{N}_{0}$ and $s \geq 2 m+4$ we have

$$
\begin{aligned}
\frac{d}{d t} \int_{\Sigma}|\phi|^{2} \gamma^{s} d \mu+\frac{3}{4} \int_{\Sigma}\left|\nabla^{2} \phi\right|^{2} \gamma^{s} d \mu \leq & \int_{\Sigma}\left(P_{3}^{m+2}(A)+P_{5}^{m}(A)\right) * \phi \gamma^{s} d \mu \\
& +c \int_{[\gamma>0]}|A|^{2} \gamma^{s-4-2 m} d \mu .
\end{aligned}
$$

Proof. We estimate the terms in (3.2). From (2.22) we have $Y=P_{3}^{m+2}(A)+$ $P_{5}^{m}(A)$, and (3.8) implies

$$
\begin{aligned}
& \int_{\Sigma} 2\langle Y, \phi\rangle \gamma^{s} d \mu+\int_{\Sigma}\langle A * \phi * \phi, V\rangle \gamma^{s} d \mu+c \int_{\Sigma}|\phi|^{2}\left(|\nabla A|^{2}+|A|^{4}\right) \gamma^{s} d \mu \\
& =\int_{\Sigma}\left(P_{3}^{m+2}(A)+P_{5}^{m}(A)\right) * \phi \gamma^{s} d \mu .
\end{aligned}
$$

By (3.9) we next have

$$
\int_{\Sigma}|\phi|^{2} \gamma^{s-1} \partial_{t} \gamma d \mu=\int_{\Sigma}|\phi|^{2} \gamma^{s-1}(D \widetilde{\gamma} \circ f)\left(\Delta H+P_{3}^{0}(A)\right) d \mu .
$$

The second term on the right is estimated by Young's inequality:

$$
\int_{\Sigma}|\phi|^{2} \gamma^{s-1}(D \widetilde{\gamma} \circ f) P_{3}^{0}(A) d \mu \leq c \int_{\Sigma}|\phi|^{2}|A|^{4} \gamma^{s} d \mu+c \int_{\Sigma}|\phi|^{2} \gamma^{s-4} d \mu .
$$

For the first term we observe, using an adapted frame, summation over $i, j$ and (2.7)

$$
\begin{aligned}
D_{e_{i}}\left((D \widetilde{\gamma} \circ f) \cdot \nabla_{e_{i}} H\right) & =\left(D^{2} \widetilde{\gamma} \circ f\right)\left(D_{e_{i}} f, \nabla_{e_{i}} H\right)+(D \widetilde{\gamma} \circ f) \cdot D_{e_{i}}\left(\nabla_{e_{i}} H\right) \\
& =\left(D^{2} \widetilde{\gamma} \circ f\right)\left(D_{e_{i}} f, \nabla_{e_{i}} H\right)+(D \widetilde{\gamma} \circ f) \Delta H
\end{aligned}
$$




$$
-\left\langle\nabla_{e_{i}} H, A\left(e_{i}, e_{j}\right)\right\rangle(D \widetilde{\gamma} \circ f) \cdot D_{e_{j}} f
$$

We thus get

$$
\begin{aligned}
& \int_{\Sigma}|\phi|^{2} \gamma^{s-1}(D \widetilde{\gamma} \circ f) \cdot \Delta H d \mu \\
& =-\int_{\Sigma} D_{e_{i}}\left(\left|\phi^{2}\right| \gamma^{s-1}\right)(D \widetilde{\gamma} \circ f) \cdot \nabla_{e_{i}} H d \mu \\
& -\int_{\Sigma}|\phi|^{2} \gamma^{s-1}\left(D^{2} \widetilde{\gamma} \circ f\right)\left(D_{e_{i}} f, \nabla_{e_{i}} H\right) d \mu \\
& +\int_{\Sigma}|\phi|^{2} \gamma^{s-1}\left\langle\nabla_{e_{i}} H, A\left(e_{i}, e_{j}\right)\right\rangle(D \widetilde{\gamma} \circ f) \cdot D_{e_{j}} f d \mu \\
& \leq c \int_{\Sigma}|\phi||\nabla \phi||\nabla A| \gamma^{s-1} d \mu+c \int_{\Sigma}|\phi|^{2}|\nabla A| \gamma^{s-2} d \mu \\
& +c \int_{\Sigma}|\phi|^{2}|\nabla A| \gamma^{s-1} d \mu+c \int_{\Sigma}|\phi|^{2}|A||\nabla A| \gamma^{s-1} d \mu \\
& \leq c \int_{\Sigma}|\nabla \phi|^{2} \gamma^{s-2} d \mu+c \int_{\Sigma}|\phi|^{2}|\nabla A|^{2} \gamma^{s} d \mu \\
& +c \int_{\Sigma}|\phi|^{2} \gamma^{s-4} d \mu+c \int_{\Sigma}|\phi|^{2}|A|^{2} \gamma^{s-2} d \mu \\
& \leq c \int_{\Sigma}|\nabla \phi|^{2} \gamma^{s-2} d \mu+c \int_{\Sigma}|\phi|^{2} \gamma^{s-4} d \mu \\
& +\int_{\Sigma}\left(P_{3}^{m+2}(A)+P_{5}^{m}(A)\right) * \phi \gamma^{s} d \mu \text {. }
\end{aligned}
$$

Now the interpolation inequality in Corollary 5.3 implies

$$
\begin{aligned}
\int_{\Sigma}|\phi|^{2} \gamma^{s-4} d \mu+\int_{\Sigma}|\nabla \phi|^{2} \gamma^{s-2} d \mu \leq & \varepsilon \int_{\Sigma}\left|\nabla^{2} \phi\right|^{2} \gamma^{s} d \mu \\
& +c(\varepsilon) \int_{[\gamma>0]}|A|^{2} \gamma^{s-4-2 m} d \mu .
\end{aligned}
$$


Collecting terms we arrive at

$$
\begin{aligned}
\int_{\Sigma}|\phi|^{2} s \gamma^{s-1} \partial_{t} \gamma d \mu \leq & \varepsilon \int_{\Sigma}\left|\nabla^{2} \phi\right|^{2} \gamma^{s} d \mu+c(\varepsilon) \int_{[\gamma>0]}|A|^{2} \gamma^{s-4-2 m} d \mu \\
& +\int_{\Sigma}\left(P_{3}^{m+2}(A)+P_{5}^{m}(A)\right) * \phi \gamma^{s} d \mu .
\end{aligned}
$$

The remaining term in (3.2) is estimated by

$$
\begin{aligned}
& \int_{\Sigma}|\phi|^{2} \gamma^{s-4}\left(|\nabla \gamma|^{4}+\gamma^{2}\left|\nabla^{2} \gamma\right|^{2}\right) d \mu \\
& \leq c \int_{\Sigma}|\phi|^{2} \gamma^{s-4} d \mu+c \int_{\Sigma}|\phi|^{2}\left(1+|A|^{2}\right) \gamma^{s-2} d \mu \\
& \leq c \int_{\Sigma}|\phi|^{2} \gamma^{s-4} d \mu+c \int_{\Sigma}|\phi|^{2}|A|^{4} \gamma^{s} d \mu,
\end{aligned}
$$

where (3.7) was used. Using again (3.11) the claim follows.

We finally state the scaled version of the proposition.

Corollary 3.4. Suppose $\chi_{B_{\varrho}\left(x_{0}\right)} \leq \tilde{\gamma} \leq \chi_{B_{2 \varrho}\left(x_{0}\right)}$ and $\left\|D^{j} \widetilde{\gamma}\right\|_{L^{\infty}} \leq c \rho^{-3}$ for $j=1,2$. Under the assumptions of Proposition 3.3, we then have

$$
\begin{aligned}
\frac{d}{d_{i}^{i}} \int_{\Sigma}|\phi|^{2} \gamma^{s} d \mu+\frac{3}{4} \int_{\Sigma}\left|\nabla^{2} \phi\right|^{2} \gamma^{s} d \mu \leq & \int_{\Sigma}\left(P_{3}^{m+2}(A)+P_{5}^{m}(A)\right) * \phi \gamma^{s} d \mu \\
& +-\frac{c}{\varrho^{4+2 m}} \int_{[\gamma>0]}|A|^{2} \gamma^{s-4-2 m} d \mu .
\end{aligned}
$$

\section{Control by concentration of curvature.}

Here we prove lecal estimates for the Willmore flow subject to the conditicin that the curvature remains small locaily in $L^{2}$. In particular we obtain the lower bourd on the lifespan depmidirg on the initial concentration stated in the introduction.

We shall need the following Soboler inequality due to Michael and Simon. Note that the immersed case of (4.1) follows trivially from the embedied 
case, by selecting an embedding $f_{0}: \Sigma \rightarrow \mathbb{R}^{m}$, applying the inequality to $\left(f, \varepsilon f_{0}\right): \Sigma \rightarrow \mathbb{R}^{n+m}$ and letting $\varepsilon \searrow 0$. Furthermore (4.1) also applies to normal-valued forms $\phi$ along $f$, due to Kato's inequality $|\nabla| \phi|| \leq|\nabla \phi|$.

Theorem 4.1 ([7]). Let $f: \Sigma \rightarrow \mathbb{R}^{n}$ be a smooth immersion. Then for any $u \in C_{c}^{1}(\Sigma)$ we have

$$
\left(\int_{\Sigma} u^{2} d \mu\right)^{\frac{1}{2}} \leq c\left(\int_{\Sigma}|\nabla u| d \mu+\int_{\Sigma}|H||u| d \mu\right),
$$

where $c=c(n)$.

Lemma 4.2. For any immersion $f: \Sigma \rightarrow \mathbb{R}^{n}$ and $\gamma \in C_{c}^{1}(\Sigma), 0 \leq \gamma \leq 1$, we have the inequality

$$
\begin{aligned}
\int_{\Sigma}|A|^{6} \gamma^{4} d \mu+\int_{\Sigma}|A|^{2}|\nabla A|^{2} \gamma^{4} d \mu \leq & c \int_{[\gamma>0]}|A|^{2} d \mu \int_{\Sigma}\left(\left|\nabla^{2} A\right|^{2} \gamma^{4}+|A|^{6} \gamma^{4}\right) d \mu \\
& +c\left(\int_{[\gamma>0]}|A|^{2} d \mu\right)^{2}
\end{aligned}
$$

where $c$ depends on $n$ and $\|\nabla \gamma\|_{L^{\infty}(\Sigma)}$.

Proof. Approximating $u=|A||\nabla A| \gamma^{2}$ and applying Theorem 4.1, we obtain

$$
\begin{aligned}
& \int_{\Sigma}|A|^{2}|\nabla A|^{2} \gamma^{4} \\
& \leq c\left(\int_{\Sigma}|A|\left|\nabla^{2} A\right| \gamma^{2} d \mu+\int_{\Sigma}|\nabla A|^{2} \gamma^{2} d \mu\right. \\
& \left.\quad+\int_{\Sigma}|A||\nabla A| 2 \gamma|\nabla \gamma| d \mu+\int_{\Sigma}|A|^{2}|\nabla A| \gamma^{2} d \mu\right)^{2} \\
& \leq c \int_{[\gamma>0]}|A|^{2} d \mu\left(\int_{\Sigma}\left|\nabla^{2} A\right|^{2} \gamma^{4} d \mu+\int_{\Sigma}|A|^{6} \gamma^{4} d \mu\right)+c\left(\int_{[\gamma>0]}|A|^{2} d \mu\right)^{2}
\end{aligned}
$$




$$
+c\left(\int_{\Sigma}|\nabla A|^{2} \gamma^{2} d \mu\right)^{2}
$$

as

$$
\left(\int_{\Sigma}|A|^{4} \gamma^{2} d \mu\right)^{2} \leq \int_{[\gamma>0]}|A|^{2} d \mu \int_{\Sigma}|A|^{6} \gamma^{4} d \mu .
$$

Integrating by parts, we estimate the last integral in (4.3) by

$$
\begin{aligned}
\int_{\Sigma}|\nabla A|^{2} \gamma^{2} d \mu \leq & \int_{\Sigma}|A|\left|\nabla^{2} A\right| \gamma^{2} d \mu+\int_{\Sigma}|A||\nabla A| 2 \gamma|\nabla \gamma| d \mu \\
\leq & \left(\int_{[\gamma>0]}|A|^{2} d \mu \int_{\Sigma}\left|\nabla^{2} A\right|^{2} \gamma^{4} d \mu\right)^{\frac{1}{2}}+c \int_{[\gamma>0]}|A|^{2} d \mu \\
& +\frac{1}{2} \int_{\Sigma}|\nabla A|^{2} \gamma^{2} d \mu .
\end{aligned}
$$

Absorbing and plugging into (4.3) yields the estimate for the second term in (4.2).

Next we apply Theorem 4.1 to $u=|A|^{3} \gamma^{2}$ and obtain

$$
\begin{aligned}
& \int_{\Sigma}|A|^{6} \gamma^{4} d \mu \\
& \leq c\left(\int_{\Sigma} 3|A|^{2}|\nabla A| \gamma^{2} d \mu+\int_{\Sigma}|A|^{3} 2 \gamma|\nabla \gamma| d \mu+\int_{\Sigma}|A|^{4} \gamma^{2} d \mu\right)^{2} \\
& \leq c\left(\int_{\Sigma}|\nabla A|^{2} \gamma^{2} d \mu\right)^{2}+c\left(\int_{\Sigma}|A|^{4} \gamma^{2} d \mu\right)^{2}+c\left(\int_{[\gamma>0]}|A|^{2} d \mu\right)^{2} .
\end{aligned}
$$

Using (4.4) and (4.5) yields the estimate for the first term in (4.2), and the proof of the lemma is complete.

In the following, we set for any normal $l$-form $\phi$ on $\Sigma$ and any measurable set $U \subseteq \Sigma$

$$
\|\phi\|_{p, U}=\left(\int_{U}|\phi|^{p} d \mu\right)^{\frac{1}{p}}
$$


Lemma 4.3. For any normal $l$-form $\phi$ on $\Sigma$ and $\gamma$ as in (3.7) we have (4.7) $\|\phi\|_{\infty,[\gamma=1]}^{4} \leq c\|\phi\|_{2,[\gamma>0]}^{2}\left(\left\|\nabla^{2} \phi\right\|_{2,[\gamma>0]}^{2}+\left\||\phi|^{2}|A|^{4}\right\|_{1,[\gamma>0]}+\|\phi\|_{2,[\gamma>0]}^{2}\right)$. Moreover, if $\phi=A$ and if

$$
\|A\|_{2,[\gamma>0]}^{2} \leq \varepsilon_{0}
$$

for some $\varepsilon_{0}$ small enough depending on the constants in (3.7), then

$$
\|A\|_{\infty,[\gamma=1]}^{4} \leq c\|A\|_{2,[\gamma>0]}^{2}\left(\left\|\nabla^{2} A\right\|_{2,[\gamma>0]}^{2}+\|A\|_{2,[\gamma>0]}^{2}\right) .
$$

Proof. We put $\psi=\phi \gamma^{2}$. Taking $m=2, p=4, \alpha=\frac{2}{3}$ in the multiplicative Sobolev inequality from Theorem 5.6 yields

$$
\|\psi\|_{\infty} \leq c\|\psi\|_{2}^{\frac{1}{3}}\left(\|\nabla \psi\|_{4}+\|\psi H\|_{4}\right)^{\frac{2}{3}} .
$$

Now using Lemma 5.1 with $\gamma \equiv 1$ we get

$$
\|\psi\|_{\infty} \leq c\|\psi\|_{2}^{\frac{1}{3}}\left(\|\psi\|_{\infty}^{\frac{1}{3}}\left\|\nabla^{2} \psi\right\|_{2}^{\frac{1}{3}}+\|\psi H\|_{4}^{\frac{2}{3}}\right) .
$$

We estimate the second term by

$$
\|\psi H\|_{4}^{\frac{2}{3}} \leq\|\psi\|_{\infty}^{\frac{1}{3}}\left\|\psi^{\frac{1}{2}} H\right\|_{4}^{\frac{2}{3}}
$$

and get

$$
\|\psi\|_{\infty}^{4} \leq c\|\psi\|_{2}^{2}\left(\left\|\nabla^{2} \psi\right\|_{2}^{2}+\left\|\psi^{2}|H|^{4}\right\|_{1}\right)
$$

Next, we note

$$
\begin{aligned}
\left\|\nabla^{2} \psi\right\|_{2}^{2} & =\int_{\Sigma}\left|\nabla^{2}\left(\phi \gamma^{2}\right)\right|^{2} d \mu \\
& \leq c \int_{\Sigma}\left|\nabla^{2} \phi\right|^{2} \gamma^{4} d \mu+c \int_{\Sigma}|\nabla \phi|^{2}\left|\nabla \gamma^{2}\right|^{2} d \mu+c \int_{\Sigma}|\phi|^{2}\left|\nabla^{2} \gamma^{2}\right|^{2} d \mu,
\end{aligned}
$$

and using (3.7) and Corollary 5.2

$$
\left\|\nabla^{2} \psi\right\|_{2}^{2} \leq c \int_{\Sigma}\left|\nabla^{2} \phi\right|^{2} \gamma^{4} d \mu+c \int_{\Sigma}|\nabla \phi|^{2} \gamma^{2} d \mu
$$




$$
\begin{aligned}
& +c \int_{\Sigma}|\phi|^{2}\left(\chi_{[\gamma>0]}+\gamma^{2}|A|^{2}\right) d \mu \\
\leq & c \int_{[\gamma>0]}\left(\left|\nabla^{2} \phi\right|^{2}+|\phi|^{2}\right) d \mu+c \int_{\Sigma}|\phi|^{2}|A|^{4} \gamma^{4} d \mu .
\end{aligned}
$$

Plugging into (4.10) yields

(4.11) $\quad\|\psi\|_{\infty}^{4} \leq c\|\psi\|_{2}^{2}\left(\left\|\nabla^{2} \phi\right\|_{2,[\gamma>0]}^{2}+\|\phi\|_{2,[\gamma>0]}^{2}+\int_{\Sigma}|\phi|^{2}|A|^{4} \gamma^{4} d \mu\right)$.

This yields (4.7).

Under the assumption (4.8), we get from (4.2) after absorbing that

$$
\begin{aligned}
\int_{\Sigma}|A|^{6} \gamma^{4} d \mu & \leq c \varepsilon_{0} \int_{\Sigma}\left|\nabla^{2} A\right|^{2} \gamma^{4} d \mu+c\|A\|_{2,[\gamma>0]}^{2} \\
& \leq c\left(\left\|\nabla^{2} A\right\|_{2,[\gamma>0]}^{2}+\|A\|_{2,[\gamma>0]}^{2}\right) .
\end{aligned}
$$

Plugging into (4.11) with $\phi=A$ yields

$$
\|A\|_{\infty,[\gamma=1]}^{4} \leq c\|A\|_{2,[\gamma>0]}^{2}\left(\left\|\nabla^{2} A\right\|_{2,[\gamma>0]}^{2}+\|A\|_{2,[\gamma>0]}^{2}\right),
$$

which is (4.9).

Proposition 4.4. Let $f: \Sigma \times[0, T] \rightarrow \mathbb{R}^{n}$ be a Willmore flow, $\gamma$ as in (3.7) and let

$$
\varepsilon=\sup _{0 \leq t \leq T}\|A\|_{2,[\gamma>0]}^{2} \leq \varepsilon_{0}
$$

for some $\varepsilon_{0}$ small enough depending on the constants in (3.7).

Then for any $t \in[0, T]$, we have

$$
\begin{aligned}
& \int_{[\gamma=1]}|A|^{2} d \mu+\frac{1}{2} \int_{0}^{t} \int_{[\gamma=1]}\left(\left|\nabla^{2} A\right|^{2}+|A|^{2}|\nabla A|^{2}+|A|^{6}\right) d \mu d \tau \\
& \leq \int_{\left[\gamma_{0}>0\right]}\left|A_{0}\right|^{2} d \mu_{0}+c \varepsilon t .
\end{aligned}
$$


Proof. We recall from Proposition 3.3 for $m=0$ and from Lemma 4.2 that

$$
\begin{aligned}
& \frac{d}{d t} \int_{\Sigma}|A|^{2} \gamma^{4} d \mu+\frac{3}{4} \int_{\Sigma}\left(\left|\nabla^{2} A\right|^{2} \gamma^{4}+|A|^{2}|\nabla A|^{2} \gamma^{4}+|A|^{6} \gamma^{4}\right) d \mu \\
& \leq c \int_{\Sigma}\left(|A|^{3}\left|\nabla^{2} A\right| \gamma^{4}+|A|^{2}|\nabla A|^{2} \gamma^{4}+|A|^{6} \gamma^{4}\right) d \mu+c \int_{[\gamma>0]}|A|^{2} d \mu \\
& \leq \frac{1}{4} \int_{\Sigma}\left|\nabla^{2} A\right|^{2} \gamma^{4} d \mu+c \int_{[\gamma>0]}|A|^{2} d \mu\left(\int_{\Sigma}\left(\left|\nabla^{2} A\right|^{2} \gamma^{4}+|A|^{6} \gamma^{4}\right) d \mu\right) \\
& \quad+c \int_{[\gamma>0]}|A|^{2} d \mu+c\left(\int_{[\gamma>0]}|A|^{2} d \mu\right)^{2} .
\end{aligned}
$$

Since $\int_{[\gamma>0]}|A|^{2} d \mu \leq \varepsilon_{0}$ by (4.12), absorbing yields

$$
\frac{d}{d t} \int_{\Sigma}|A|^{2} \gamma^{4} d \mu+\frac{1}{2} \int_{\Sigma}\left(\left|\nabla^{2} A\right|^{2} \gamma^{4}+|A|^{2}|\nabla A|^{2} \gamma^{4}+|A|^{6} \gamma^{4}\right) d \mu \leq c \varepsilon .
$$

Now (4.13) follows by integration over $[0, t]$.

Proposition 4.5. Let $f: \Sigma \times I \rightarrow \mathbb{R}^{n}$ be a Willmore flow. Then for $\phi=$ $\nabla^{m} A, m \in \mathbb{N}_{0}$ and $\gamma$ as in (3.7) we have for any $s \geq 2 m+4$

$$
\begin{aligned}
\frac{d}{d t} \int|\phi|^{2} \gamma^{s} d \mu+\frac{1}{2} \int_{\Sigma}\left|\nabla^{2} \phi\right|^{2} \gamma^{s} d \mu \leq & c\|A\|_{\infty,[\gamma>0]}^{4} \int_{\Sigma}|\phi|^{2} \gamma^{s} d \mu \\
& +c\left(1+\|A\|_{\infty,[\gamma>0]}^{4}\right) \cdot\|A\|_{2,[\gamma>0]}^{2}
\end{aligned}
$$

Proof. By Proposition 3.3, it suffices to prove that

$$
\begin{aligned}
& \int_{\Sigma}\left(P_{3}^{m+2}(A)+P_{5}^{m}(A)\right) * \phi \gamma^{s} d \mu \\
& \leq \frac{1}{4} \int_{\Sigma}\left|\nabla^{2} \phi\right|^{2} \gamma^{s} d \mu+c\|A\|_{\infty,[\gamma>0]}^{4} \cdot \int_{\Sigma}|\phi|^{2} \gamma^{s} d \mu \\
& \quad+c\left(1+\|A\|_{\infty,[\gamma>0]}^{4}\right) \cdot\|A\|_{2,[\gamma>0]}^{2} .
\end{aligned}
$$


We estimate the second term by choosing $r=6, k=m$ in Corollary 5.5 and get

$$
\left|\int_{\Sigma} P_{5}^{m}(A) * \phi \gamma^{s} d \mu\right| \leq c\|A\|_{\infty,[\gamma>0]}^{4}\left(\int_{\Sigma}|\phi|^{2} \gamma^{s} d \mu+\|A\|_{2,[\gamma>0]}^{2}\right) .
$$

Applying Corollary 5.5 with $r=4, k=m+1$ further yields

$$
\begin{aligned}
& \left|\int_{\Sigma} P_{3}^{m+2}(A) \phi \gamma^{s} d \mu\right| \\
& \leq\left|\int_{\Sigma} \nabla^{m+2} A * A * A * \nabla^{m} A \cdot \gamma^{s} d \mu\right| \\
& \quad+c\|A\|_{\infty,[\gamma>0]}^{2}\left(\int_{\Sigma}|\nabla \phi|^{2} \gamma^{s} d \mu+\|A\|_{2,[\gamma>0]}^{2}\right) \\
& \leq \tau \int_{\Sigma}\left|\nabla^{2} \phi\right|^{2} \gamma^{s} d \mu+c(\tau)\|A\|_{\infty,[\gamma>0]}^{4} \cdot \int_{\Sigma}|\phi|^{2} \gamma^{s} d \mu \\
& \quad+c\|A\|_{\infty,[\gamma>0]}^{2}\|A\|_{2,[\gamma>0]}^{2}+c\|A\|_{\infty,[\gamma>0]}^{2} \int_{\Sigma}|\nabla \phi|^{2} \gamma^{s} d \mu .
\end{aligned}
$$

Using Lemma 5.1 with $p=q=2 r=2, \alpha=0, \beta=1, t=0$, we estimate the last term by

$$
\begin{aligned}
& \|A\|_{\infty,[\gamma>0]}^{2} \int_{\Sigma}|\nabla \phi|^{2} \gamma^{s} d \mu \\
& \leq c\|A\|_{\infty,[\gamma>0]}^{2}\left(\int_{\Sigma}|\phi|^{2} \gamma^{s} d \mu\right)^{\frac{1}{2}}\left(\int_{\Sigma}\left|\nabla^{2} \phi\right|^{2} \gamma^{s} d \mu\right)^{\frac{1}{2}} \\
& \quad+c\|A\|_{\infty,[\gamma>0]}^{2}\left(\int_{\Sigma}|\phi|^{2} \gamma^{s} d \mu\right)^{\frac{1}{2}} \cdot\left(\int_{\Sigma}|\nabla \phi|^{2} \gamma^{s-2} d \mu\right)^{\frac{1}{2}} \\
& \leq \tau \int_{\Sigma}\left|\nabla^{2} \phi\right|^{2} \gamma^{s} d \mu+c(\tau)\|A\|_{\infty,[\gamma>0]}^{4} \cdot \int_{\Sigma}|\phi|^{2} \gamma^{s} d \mu \\
& \quad+c \int_{\Sigma}|\nabla \phi|^{2} \gamma^{s-2} d \mu .
\end{aligned}
$$


Finally, since $s-2 \geq(m+1) 2$, we may use Corollary 5.3 with $k=m+1$ to get

$$
\begin{aligned}
\int_{\Sigma}|\nabla \phi|^{2} \gamma^{s-2} d \mu & =\int_{\Sigma}\left|\nabla^{m+1} A\right|^{2} \gamma^{s-2} d \mu \\
& \leq \tau \int_{\Sigma}\left|\nabla^{2} \phi\right|^{2} \gamma^{s} d \mu+c(\tau) \int_{[\gamma>0]}|A|^{2} d \mu
\end{aligned}
$$

concluding the proof of (4.15), hence of the proposition.

Proposition 4.6. Let $f: \Sigma \times[0, T] \rightarrow \mathbb{R}^{n}$ be a Willmore flow and $\gamma$ as in (3.7). If

$$
\sup _{0 \leq t \leq T} \int_{[\gamma>0]}|A|^{2} d \mu \leq \varepsilon_{0}
$$

where $\varepsilon_{0}$ is small enough depending on the constants in (3.7), then

$$
\left\|\nabla^{m} A\right\|_{\infty,[\gamma=1]} \leq c\left(m, T, \alpha_{0}(m+2)\right),
$$

where $\alpha_{0}(m)=\sum_{j=0}^{m}\left\|\nabla^{j} A_{0}\right\|_{2,\left[\gamma_{0}>0\right]}$.

Proof. For $0 \leq \sigma<\tau \leq 1$ we use cutoff functions $\gamma_{\sigma, \tau}=\psi_{\sigma, \tau} \circ \gamma$ satisfying $\gamma_{\sigma, \tau}=0$ for $\gamma \leq \sigma$ and $\gamma_{\sigma, \tau}=1$ for $\gamma \geq \tau$. With $\sigma=0, \tau=\frac{1}{2}$ we infer from(4.13) that

$$
\int_{0}^{T} \int_{\left[\gamma \geq \frac{1}{2}\right]}\left(\left|\nabla^{2} A\right|^{2}+|A|^{6}\right) d \mu \leq c \varepsilon_{0}(1+T) .
$$

Next we let $\sigma=\frac{1}{2}, \tau=\frac{3}{4}$ and get from (4.9)

$$
\int_{0}^{T}\|A\|_{\infty,\left[\gamma \geq \frac{3}{4}\right]}^{4} d t \leq c \varepsilon_{0}\left(c \varepsilon_{0}(1+T)+\varepsilon_{0} T\right) \leq c \varepsilon_{0}^{2}(1+T) .
$$

Now (4.14) with $\sigma=\frac{3}{4}, \tau=\frac{7}{8}$ yields for $\phi=\nabla^{m} A, s=2 m+4$ and $0 \leq t \leq T$ the inequality

$$
\int_{\Sigma}|\phi|^{2} \gamma_{\sigma, \tau}^{s} d \mu+\int_{0}^{t} \int_{\left[\gamma \geq \frac{7}{8}\right]}\left|\nabla^{2} \phi\right|^{2} d \mu d t^{\prime}
$$




$$
\begin{aligned}
\leq & \int_{\Sigma}\left|\phi_{0}\right|^{2}\left(\gamma_{0}\right)_{\sigma, \tau}^{s} d \mu_{0}+c(m) \varepsilon_{0}\left(T+\int_{0}^{T}\|A\|_{\infty,\left[\gamma \geq \frac{3}{4}\right]}^{4} d t\right) \\
& +c(m) \int_{0}^{t}\|A\|_{\infty,\left[\gamma \geq \frac{3}{4}\right]}^{4}\left(\int_{\Sigma}|\phi|^{2} \gamma_{\sigma, \tau}^{s} d \mu\right) d t^{\prime} .
\end{aligned}
$$

Using Gronwall's Lemma and (4.17) we obtain

$$
\begin{aligned}
& \sup _{0 \leq t \leq T} \int_{\left[\gamma \geq \frac{7}{8}\right]}\left|\nabla^{m} A\right|^{2} d \mu+\int_{0}^{T} \int_{\left[\gamma \geq \frac{7}{8}\right]}\left|\nabla^{m+2} A\right|^{2} d \mu d t \\
& \leq c(m, T)\left(1+\int_{\left[\gamma_{0}>0\right]}\left|\nabla^{m} A_{0}\right|^{2} d \mu_{0}\right)=c\left(m, T, \alpha_{0}(m)\right) .
\end{aligned}
$$

From this and (4.9) we deduce

$$
\|A\|_{\infty,\left[\gamma \geq \frac{15}{16}\right]}^{4} \leq c \varepsilon_{0}\left(c\left(2, T, \alpha_{0}(2)\right)+\varepsilon_{0}\right)=c\left(T, \alpha_{0}(2)\right) .
$$

Finally, using (4.7) for $\phi=\nabla^{m} A$ we get

$$
\begin{aligned}
& \left\|\nabla^{m} A\right\|_{\infty,[\gamma=1]}^{4} \\
& \leq c\left(m, T, \alpha_{0}(m)\right)\left(\left(c\left(m, T, \alpha_{0}(m+2)\right)+\|A\|_{\infty,\left[\gamma \geq \frac{15}{16}\right]}^{4} c\left(m, T, \alpha_{0}(m)\right)\right.\right. \\
& \left.\quad+c\left(m, T, \alpha_{0}(m)\right)\right) \\
& \leq c\left(m, T, \alpha_{0}(m+2)\right) .
\end{aligned}
$$

In slight abuse of notation (4.6), we write for $V \subseteq \mathbb{R}^{n}$ and any normal $l$-form $\phi$ on $\Sigma$

$$
\|\phi\|_{p, V}=\left(\int_{f^{-1}(V)}|\phi|^{p} d \mu\right)^{\frac{1}{p}} .
$$

We are ready to prove our main theorem.

Proof of Theorem 1.2. Rescaling, we may assume that $\varrho=1$. Put

$$
\varepsilon(t)=\sup _{x \in \mathbb{R}^{n}} \int_{B_{1}(x)}|A|^{2} d \mu .
$$


By a trivial covering argument, we get for some $\Gamma=\Gamma(n)>1$

$$
\varepsilon(t) \leq \Gamma \cdot \sup _{x \in \mathbb{R}^{n}} \int_{B_{\frac{1}{2}}(x)}|A|^{2} d \mu .
$$

The function $\varepsilon:[0, T) \rightarrow \mathbb{R}$ is continuous by compactness of $f(\Sigma \times[0, t])$ for $t<T$. Now let $\lambda>0$ be a parameter, and define

$$
t_{0}:=\sup \{0 \leq t \leq \min (T, \lambda): \varepsilon(\tau) \leq 3 \Gamma \varepsilon \text { for } 0 \leq \tau<t\} .
$$

By continuity of $\varepsilon(t)$ we have $t_{0}>0$; furthermore

$$
\varepsilon\left(t_{0}\right)=3 \Gamma \varepsilon \quad \text { if } t_{0}<\min (T, \lambda) .
$$

Fix a cutoff function $\tilde{\gamma} \in C^{2}\left(\mathbb{R}^{n}\right)$ with $\|\widetilde{\gamma}\|_{C^{2}\left(\mathbb{R}^{n}\right)} \leq C(n)$ and $\chi_{B_{1 / 2}(x)} \leq$ $\widetilde{\gamma} \leq \chi_{B_{1}(x)}$; then $\gamma=\widetilde{\gamma} \circ f$ satisfies (3.7) for constants depending only on $n$. Definition 4.19 implies condition (4.12) of Proposition 4.4 on $\left[0, t_{0}\right)$. Therefore

$$
\int_{B_{\frac{1}{2}}(x)}|A|^{2} d \mu \leq \int_{B_{1}(x)}\left|A_{0}\right|^{2} d \mu_{0}+c \Gamma \varepsilon t \leq 2 \varepsilon \quad \text { for } 0 \leq t<t_{0},
$$

if we take $\lambda=(c \Gamma)^{-1}$. From (4.18) we conclude that

$$
\varepsilon(t) \leq 2 \Gamma \varepsilon \text { for } 0 \leq t<t_{0},
$$

and thus (4.20) implies $t_{0}=\min \left(T,(c \Gamma)^{-1}\right)$. Now if $t_{0}=(c \Gamma)^{-1}$, then (1.5) holds and (4.21) implies (1.6). Therefore the theorem will be proved, if we lead the assumption

$$
t_{0}=T
$$

to a contradiction. First by $(4.21)$ and $T=t_{0} \leq(c \Gamma)^{-1}$ we can apply Proposition 4.6 to obtain

$$
\left\|\nabla^{m} A\right\|_{\infty} \leq c\left(n, m, f_{0}\right) .
$$

According to (2.15) and the Willmore flow equation, the time derivative of the metric satisfies

$$
h=\partial_{t} g=-2\left\langle A, \partial_{t} f\right\rangle=\nabla^{2} A * A+A * A * A * A .
$$


Therefore (4.23) implies

$$
\left\|\nabla^{m} h\right\|_{\infty} \leq c\left(n, m, f_{0}\right) .
$$

Likewise, (2.17) yields for the derivative of the Levi-Cività connection, i.e., the Christoffel symbols

$$
\partial_{t} \nabla=\nabla^{3} A * A+\nabla^{2} A * \nabla A+\nabla A * A * A * A .
$$

Again by (4.23)

$$
\left\|\nabla^{m}\left(\partial_{t} \nabla\right)\right\|_{\infty} \leq c\left(n, m, f_{0}\right) .
$$

Lemma 14.2 in [3] shows that the metrics $g(t), 0 \leq t<T$ are equivalent. We now take a local chart $\psi: U \longrightarrow \Sigma$ such that

$$
\frac{1}{c\left(n, f_{0}\right)} \leq\left(g_{i j}(t)\right) \leq c\left(n, f_{0}\right) \quad \text { on } U, 0 \leq t<T .
$$

Let $\Gamma$ be the associated Christoffel symbols and denote the coordinate derivative by $\partial$. For any tensor $T$ we have the formula

$$
\nabla^{m} T=\partial^{m} T+\sum_{l=1}^{m} \sum_{k_{1}+\ldots+k_{l}+k \leq m-1} \partial^{k_{1}} \Gamma \cdot \ldots \cdot \partial^{k_{l}} \Gamma \cdot \partial^{k} T .
$$

This is immediate for $m=1$ and follows then by induction. Therefore letting $\gamma_{m}=|\Gamma|+\ldots+\left|\partial^{m} \Gamma\right|$ we have

$$
\left|\partial^{m} T\right| \leq c\left(n, m, \gamma_{m-1}\right)\left(\left|\nabla^{m} T\right|+\left|\partial^{m-1} T\right|+\ldots+|T|\right) .
$$

Hence by induction

$$
\left|\partial^{m} T\right| \leq c\left(n, m, \gamma_{m-1}\right)\left(\left|\nabla^{m} T\right|+\left|\nabla^{m-1} T\right|+|T|\right) .
$$

We apply this to $T=\partial_{t} \Gamma$. Using induction and (4.25) we get

$$
\left|\partial^{m}\left(\partial_{t} \Gamma\right)\right| \leq c\left(n, m, f_{0}\right), \quad\left|\partial^{m} \Gamma\right| \leq c\left(n, m, f_{0}\right) .
$$

Turning to the derivatives of $A$ and $f$, we claim that

$$
\left\|\partial^{k} \nabla^{l} A\right\|_{\infty},\left\|\partial^{m+1} f\right\|_{\infty} \leq c\left(n, m, f_{0}\right), \quad \text { for } k+l=m \geq 0 .
$$

Clearly, this holds for $m=0$ by (4.23) and since $\|\partial f\|=1$. 
From (2.7), we obtain for $k+l=m+1$ that

$$
\begin{aligned}
\partial^{k} \nabla^{l} A-\nabla^{m+1} A & =\partial^{k} \nabla^{l} A-\nabla^{k} \nabla^{l} A \\
& =\sum_{j=1}^{k}\left(\partial^{j} \nabla^{k+l-j}-\partial^{j-1} \nabla^{k+l+1-j}\right) A \\
& =\sum_{j=1}^{k} \partial^{j-1}(\partial-\nabla) \nabla^{k+l-j} A \\
& =\sum_{j=1}^{k} \partial^{j-1}\left(\nabla^{k+l-j} A * A * \partial f\right) .
\end{aligned}
$$

Now $\partial^{i} \nabla^{k+l-j} A, \partial^{i} A$ and $\partial^{i+1} f$ for $0 \leq i \leq j-1 \leq k-1 \leq m$ are bounded by induction hypothesis, and the first part of (4.27) is proved. By the Gauß-Weingarten equations, we see that

$$
\partial^{2} f=A+\partial f \cdot \Gamma,
$$

and the second estimate in (4.27) follows from the first and (4.26).

Since $\partial_{t} f=-\mathbf{W}(f)=-\Delta H+A * A * A$, we get from (4.27) that

$$
\left\|\partial^{m} f\right\|_{\infty},\left\|\partial^{m} \partial_{t} f\right\|_{\infty} \leq c\left(n, m, f_{0}\right) .
$$

Then $f(t)$ converges in $C^{m}(\Sigma)$ as $t \nearrow T$ to a smooth function $f_{T}: \Sigma \rightarrow \mathbb{R}^{n}$. $f_{T}$ is an immersion as the metrics $g(t) \rightarrow g(T)$ are uniformly equivalent. By short time existence, we can extend the flow $f$ to an interval $[0, T+$ $\delta$ ), contradicting the maximality of $T$, hence contradicting (4.22), and the thecrem is proved.

\section{Appendix: Interpolation inequalities.}

Here we assume for all results except Theorem 5.6 that $\Sigma$ is a $d$-dimensional Riemannian manifold and that $\gamma \in C_{c}^{1}(\Sigma)$ satisfies

$$
0 \leq \gamma \leq 1, \quad|\nabla \gamma| \leq \Lambda .
$$

Our aim is to prove localized versions of the inequalities from $\S 12$ in [3]. We start with the following lemma based on partial integration. 
Lemma 5.1. Let $\frac{1}{p}+\frac{1}{q}=\frac{1}{r}, 1 \leq p, q, r \leq \infty$ and $\alpha+\beta=1, \alpha, \beta \geq 0$. For $s \geq \max \{\alpha q, \beta p\}$ and $-\frac{1}{p} \leq t \leq \frac{1}{q}$ we have

$$
\begin{aligned}
\left(\int_{\Sigma}|\nabla \phi|^{2 r} \gamma^{s} d \mu\right)^{\frac{1}{r}} \leq & c\left(\int_{[\gamma>0]}|\phi|^{q} \gamma^{s(1-t q)} d \mu\right)^{\frac{1}{q}}\left(\int_{\Sigma}\left|\nabla^{2} \phi\right|^{p} \gamma^{s(1+t p)} d \mu\right)^{\frac{1}{p}} \\
& +c \Lambda s\left(\int_{[\gamma>0]}|\phi|^{q} \gamma^{s-\alpha q} d \mu\right)^{\frac{1}{q}}\left(\int_{\Sigma}|\nabla \phi|^{p} \gamma^{s-\beta p} d \mu\right)^{\frac{1}{p}}
\end{aligned}
$$

where $c=c(d, r)$.

Proof. Using integration by parts, we get

$$
\begin{aligned}
& \int_{\Sigma}|\nabla \phi|^{2 r} \gamma^{s} d \mu \\
& \leq c \int_{\Sigma}|\phi||\nabla \phi|^{2 r-2}\left|\nabla^{2} \phi\right| \gamma^{s} d \mu+c \Lambda s \int_{\Sigma}|\phi||\nabla \phi|^{2 r-2}|\nabla \phi| \gamma^{s-1} d \mu \\
& \leq c\left(\int_{[\gamma>0]}|\phi|^{q} \gamma^{s(1-t q)} d \mu\right)^{\frac{1}{q}}\left(\int_{\Sigma}|\nabla \phi|^{2 r} \gamma^{s}\right)^{\frac{r-1}{r}}\left(\int_{\Sigma}\left|\nabla^{2} \phi\right|^{p} \gamma^{s(1+t p)}\right)^{\frac{1}{p}} \\
& \quad+c \Lambda s\left(\int_{[\gamma>0]}|\phi|^{q} \gamma^{s-\alpha q} d \mu\right)^{\frac{1}{q}}\left(\int_{\Sigma}|\nabla \phi|^{2 r} \gamma^{s} d \mu\right)^{\frac{r-1}{r}}\left(\int_{\Sigma}|\nabla \phi|^{p} \gamma^{s-\beta p} d \mu\right)^{\frac{1}{p}}
\end{aligned}
$$

since $\frac{1}{q}+\frac{r-1}{r}+\frac{1}{p}=1$.

Corollary 5.2. For $2 \leq p<\infty$ and $s \geq p$ we have

$$
\left(\int_{\Sigma}|\nabla \phi|^{p} \gamma^{s} d \mu\right)^{\frac{1}{p}} \leq \varepsilon\left(\int_{\Sigma}\left|\nabla^{2} \phi\right|^{p} \gamma^{s+p} d \mu\right)^{\frac{1}{p}}+c_{\varepsilon}\left(\int_{[\gamma>0]}|\phi|^{p} \gamma^{s-p} d \mu\right)^{\frac{1}{p}}
$$

where $c_{\varepsilon}=c_{\varepsilon}(d, p, s, \Lambda)$. 
Proof. We take $p=q=2 r, \alpha=1, \beta=0$ and $t=\frac{1}{s}$ in the previous lemma and obtain

$$
\begin{aligned}
\left(\int_{\Sigma}|\nabla \phi|^{p} \gamma^{s} d \mu\right)^{\frac{1}{p}} \leq & c\left(\int_{[\gamma>0]}|\phi|^{p} \gamma^{s-p} d \mu\right)^{\frac{1}{2 p}}\left(\int_{\Sigma}\left|\nabla^{2} \phi\right|^{p} \gamma^{s+p} d \mu\right)^{\frac{1}{2 p}} \\
& +c s\left(\int_{[\gamma>0]}|\phi|^{p} \gamma^{s-p} d \mu\right)^{\frac{1}{2 p}}\left(\int_{\Sigma}|\nabla \phi|^{p} \gamma^{s} d \mu\right)^{\frac{1}{2 p}} .
\end{aligned}
$$

Corollary 5.3. For $2 \leq p<\infty, k \in \mathbb{N}, s \geq k p$ and $c_{\varepsilon}=c_{\varepsilon}(d, p, s, k, \Lambda)$ we have

$$
\begin{aligned}
& \left(\int_{\Sigma}\left|\nabla^{k} \phi\right|^{p} \gamma^{s} d \mu\right)^{\frac{1}{p}} \\
& \leq \varepsilon\left(\int_{\Sigma}\left|\nabla^{k+1} \phi\right|^{p} \gamma^{s+p} d \mu\right)^{\frac{1}{p}}+c_{\varepsilon}\left(\int_{[\gamma>0]}|\phi|^{p} \gamma^{s-k p} d \mu\right)^{\frac{1}{p}} .
\end{aligned}
$$

Proof. For $k=1$ the statement holds by Corollary 5.2. Again by 5.2, we have

$$
\begin{aligned}
& \left(\int_{\Sigma}\left|\nabla^{k+1} \phi\right|^{p} \gamma^{s} d \mu\right)^{\frac{1}{p}} \\
& \leq \varepsilon\left(\int_{\Sigma}\left|\nabla^{k+2} \phi\right|^{p} \gamma^{s+p} d \mu\right)^{\frac{1}{p}}+c_{\varepsilon}\left(\int_{\Sigma}\left|\nabla^{k} \phi\right|^{p} \gamma^{s-p} d \mu\right)^{\frac{1}{p}} .
\end{aligned}
$$

The inductive hypothesis, applied with $s-p$ instead of $s$, yields

$$
\begin{aligned}
& \left(\int_{\Sigma}\left|\nabla^{k} \phi\right|^{p} \gamma^{s-p} d \mu\right)^{\frac{1}{p}} \\
& \leq \tau\left(\int_{\Sigma}\left|\nabla^{k+1} \phi\right|^{p} \gamma^{s} d \mu\right)^{\frac{1}{p}}+c(\tau)\left(\int_{[\gamma>0]}|\phi|^{p} \gamma^{s-(k+1) p} d \mu\right)^{\frac{1}{p}},
\end{aligned}
$$

where $c(\tau)=c(\tau, s, p, s-p, k)$. Combining the inequalities proves the result. 
Theorem 5.4. For $k \in \mathbb{N}, 1 \leq i \leq k$ and $s \geq 2 k$ we have the inequality

$$
\begin{aligned}
& \left(\int_{\Sigma}\left|\nabla^{i} \phi\right|^{\frac{2 k}{i}} \gamma^{s} d \mu\right)^{\frac{i}{2 k}} \\
& \leq c\|\phi\|_{\infty}^{1-\frac{i}{k}}\left(\left(\int_{\Sigma}\left|\nabla^{k} \phi\right|^{2} \gamma^{s} d \mu\right)^{\frac{1}{2}}+\|\phi\|_{2,[\gamma>0]}\right)^{\frac{i}{k}}
\end{aligned}
$$

where $c$ depends on $k, d, s$ and $\Lambda$.

Proof. For $1 \leq i \leq k$ we put

$$
\begin{gathered}
a_{i}=\left(\int_{\Sigma}\left|\nabla^{i} \phi\right|^{\frac{2 k}{i}} \gamma^{s} d \mu\right)^{\frac{i}{2 k}}, a_{0}=\|\phi\|_{\infty}, \\
b_{i}=\left(\int_{[\gamma>0]}|\phi|^{\frac{2 k}{i}} d \mu\right)^{\frac{i}{2 k}}, \quad b_{0}=\|\phi\|_{\infty} .
\end{gathered}
$$

Applying Lemma 5.1 for $r=\frac{k}{i}, p=\frac{2 k}{i+1}, q=\frac{2 k}{i-1}, t=\alpha=0$ and $\beta=1$ we obtain for $s \geq 2 k$

$$
a_{i}^{2} \leq c a_{i-1}\left[a_{i+1}+\left(\int_{\Sigma}\left|\nabla^{i} \phi\right|^{\frac{2 k}{i+1}} \gamma^{s-\frac{2 k}{i+1}} d \mu\right)^{\frac{i+1}{2 k}}\right] .
$$

On the other hand Corollary 5.3 yields for $s \geq 2 k$

$$
\begin{aligned}
\left(\int_{\Sigma}\left|\nabla^{i} \phi\right|^{\frac{2 k}{i+1}} \gamma^{s-\frac{2 k}{i+1}} d \mu\right)^{\frac{i+1}{2 k}} \leq & c\left(\int_{\Sigma}\left|\nabla^{i+1} \phi\right|^{\frac{2 k}{i+1}} \gamma^{s} d \mu\right)^{\frac{i+1}{2 k}} \\
& +c\left(\int_{[\gamma>0]}|\phi|^{\frac{2 k}{i+1}} \gamma^{s-\frac{2 k}{i+1}-i \frac{2 k}{i+1}} d \mu\right)^{\frac{i+1}{2 k}} \\
\leq & c\left(a_{i+1}+b_{i+1}\right) .
\end{aligned}
$$

Since $b_{i}^{2} \leq b_{i-1} b_{i+1}$ by Hölder, we obtain

$$
\left(a_{i}+b_{i}\right)^{2} \leq c\left(a_{i-1}+b_{i-1}\right)\left(a_{i+1}+b_{i+1}\right) \quad(1 \leq i \leq k-1) .
$$

Now a simple convexity argument, see Corollary 12.5 in [3], implies

$$
a_{i} \leq a_{i}+b_{i}
$$




$$
\begin{aligned}
& \leq c\left(a_{0}+b_{0}\right)^{1-\frac{i}{k}}\left(a_{k}+b_{k}\right)^{\frac{i}{k}} \\
& \leq c\|\phi\|_{\infty}^{1-\frac{i}{k}}\left(\left(\int_{\Sigma}\left|\nabla^{k} \phi\right|^{2} \gamma^{s} d \mu\right)^{\frac{1}{2}}+\|\phi\|_{2,[\gamma>0]}\right)^{\frac{i}{k}} .
\end{aligned}
$$

Corollary 5.5. Let $0 \leq i_{1}, \ldots, i_{r} \leq k, i_{1}+\ldots+i_{r}=2 k$ and $s \geq 2 k$. Then we have

$$
\left|\int_{\Sigma} \nabla^{i_{1}} \phi * \ldots * \nabla^{i_{r}} \phi \gamma^{s} d \mu\right| \leq c\|\phi\|_{\infty}^{r-2}\left(\int_{\Sigma}\left|\nabla^{k} \phi\right|^{2} \gamma^{s} d \mu+\|\phi\|_{2,[\gamma>0]}^{2}\right),
$$

where $c=c(k, d, r, s, \Lambda)$.

Proof. Assume $i_{1}, \ldots, i_{l} \geq 1$ and $i_{l+1}, \ldots, i_{r}=0$. Then by Hölder's inequality and by 5.4 we have

$$
\begin{aligned}
& \int_{\Sigma} \nabla^{i_{1}} \phi * \ldots * \nabla^{i_{r}} \phi \gamma^{s} d \mu \\
& \leq\|\phi\|_{\infty}^{r-l} \prod_{j=1}^{l}\left(\int_{\Sigma}\left|\nabla^{i_{j}} \phi\right|^{\frac{2 k}{i_{j}}} \gamma^{s} d \mu\right)^{\frac{i_{j}}{2 k}} \\
& \leq c\|\phi\|_{\infty}^{r-l} \prod_{j=1}^{l}\left[\|\phi\|_{\infty}^{1-\frac{i_{j}}{k}}\left(\left(\int_{\Sigma}\left|\nabla^{k} \phi\right|^{2} \gamma^{s} d \mu\right)^{\frac{1}{2}}+\|\phi\|_{2,[\gamma>0]}\right)^{\frac{i_{j}}{k}}\right] \\
& \leq c\|\phi\|_{\infty}^{r-2}\left(\int_{\Sigma}\left|\nabla^{k} \phi\right|^{2} \gamma^{s} d \mu+\|\phi\|_{2,[\gamma>0]}^{2}\right) .
\end{aligned}
$$

For convenience of the reader we finally include a proof of the multiplicative Sobolev inequality used in Lemma 4.3.

Theorem 5.6. Let $f: \Sigma \longrightarrow \mathbb{R}^{n}$ be a smooth, immersed surface. For $u \in C_{c}^{1}(\Sigma), 2<p \leq \infty, 0 \leq m \leq \infty$ and $0<\alpha \leq 1$ with $\frac{1}{\alpha}=\left(\frac{1}{2}-\frac{1}{p}\right) m+1$ we have

$$
\|u\|_{\infty} \leq c\|u\|_{m}^{1-\alpha}\left(\|\nabla u\|_{p}+\|H u\|_{p}\right)^{\alpha},
$$

where $c=c(n, m, p)$. 
Proof. The argument follows [6]. We may assume that $u$ is nonnegative and that

$$
c_{n}\left(\|\nabla u\|_{p}+\|H u\|_{p}\right)=1,
$$

where $c_{n}$ is the constant in the Michael-Simon Sobolev inequality (4.1). Letting $q=\frac{p}{p-1} \in[1,2)$ we infer for any $\tau \geq 0$

$$
\begin{aligned}
\left\|u^{1+\tau}\right\|_{2} & \leq c_{n}\left(\left\|\nabla\left(u^{1+\tau}\right)\right\|_{1}+\left\|H u^{1+\tau}\right\|_{1}\right) \\
& \leq c_{n}\left\|u^{\tau}\right\|_{q}\left((1+\tau)\|\nabla u\|_{p}+\|H u\|_{p}\right) \\
& \leq(1+\tau)\left\|u^{\tau}\right\|_{q},
\end{aligned}
$$

where (5.8) was used in the last step. With $k=\frac{2}{q} \in(1,2]$ we rewrite this as

$$
\|u\|_{k(1+\tau) q} \leq(1+\tau)^{\frac{1}{1+\tau}}\|u\|_{\tau q}^{\frac{\tau}{\tau+1}}
$$

Putting $\tau_{0}=\frac{m}{q} \in\left(\frac{m}{2}, m\right], \tau_{\nu+1}=k\left(1+\tau_{\nu}\right), \varepsilon_{\nu}=\frac{\tau_{\nu}}{\tau_{\nu}+1}$ and $c_{\nu}=\left(1+\tau_{\nu}\right)^{\frac{1}{1+\tau_{\nu}}}$ we obtain for $\nu \in \mathbb{N}_{0}$

$$
\|u\|_{\tau_{\nu+1} q} \leq c_{\nu}\|u\|_{\tau_{\nu} q}^{\varepsilon_{\nu}}
$$

where

$$
1+\tau_{\nu}=k^{\nu} \tau_{0}+\sum_{\mu=0}^{\nu} k^{\mu}
$$

By induction (5.9) implies

$$
\|u\|_{\tau_{\nu} q} \leq\left(\prod_{\mu=0}^{\nu-1} c_{\mu}^{\varepsilon_{\mu+1} \cdot \ldots \cdot \varepsilon_{\nu-1}}\right)\|u\|_{m}^{\varepsilon_{0} \cdot \varepsilon_{1} \cdot \ldots \cdot \varepsilon_{\nu-1}} .
$$

Now (5.10) yields

$$
\frac{1}{c} k^{\nu} \leq 1+\tau_{\nu} \leq c k^{\nu} \quad \text { for } c=c(m, p),
$$

and thus using $\varepsilon_{\mu} \leq 1$ we can estimate

$$
\log \prod_{\mu=0}^{\nu} c_{\mu}^{\varepsilon_{\mu+1} \cdot \ldots \cdot \varepsilon_{\nu}} \leq \sum_{\mu=0}^{\nu} \frac{1}{1+\tau_{\mu}} \log \left(1+\tau_{\mu}\right)
$$




$$
\leq \sum_{\mu=0}^{\infty} c k^{-\mu}(\log c+\mu \log k)=c(m, p)<\infty
$$

Using $\tau_{\nu+1}=k\left(1+\tau_{\nu}\right)$ we get from $(5.10)$

$$
\prod_{\mu=0}^{\nu} \varepsilon_{\mu}=k^{\nu} \frac{\tau_{0}}{1+\tau_{\nu}} \stackrel{\nu \rightarrow \infty}{\longrightarrow} \frac{\tau_{0}}{\tau_{0}+\frac{k}{k-1}}=1-\alpha
$$

Thus we may let $\nu \rightarrow \infty$ in (5.11) and conclude, using again (5.8),

$$
\begin{aligned}
\|u\|_{\infty} & \leq c(m, p)\|u\|_{m}^{1-\alpha} \\
& =c(m, p) c_{n}^{\alpha}\|u\|_{m}^{1-\alpha}\left(\|\nabla u\|_{p}+\|H u\|_{p}\right)^{\alpha} .
\end{aligned}
$$

\section{References.}

[1] R. Bryant, A Duality Theorem for Willmore Surfaces, J. Differential Geom., 20 (1984), 23-53.

[2] J. Escher, U. Mayer, and G. Simonett, The Surface Diffusion Flow for Immersed Hypersurfaces, Siam. J. Math. Anal., 29(6) (1998), 14191433.

[3] R. Hamilton, Three-Manifolds with Positive Ricci Curvature, J. Differential Geom., 17 (1982), 255-306.

[4] R. Kusner, Estimates for the Biharmonic Energy on Unbounded Planar Domains, and the Existence of Surfaces of Every Genus that Minimize the Squared-Mean-Curvature Integral, in 'Elliptic and Parabolic Methods in Geometry,' AK Peters, 1996.

[5] E. Kuwert and R. Schätzle, The Willmore Flow with small initial energy, (2002), to appear in Journal of Differential Geomerty.

[6] O.A. Ladyzhenskaya, V.A. Solonnikov, and N.N. Uraltseva, Linear and Quasilinear Equations of Parabolic Type, American Math. Society, 1968. 
[7] J.H. Michael and L. Simon, Sobolev and Mean-Value Inequalities on Generalized Submanifolds of $\mathbb{R}^{n}$, Comm. Pure Appl. Math., 26 (1973), 361-379.

[8] A. Polden, Closed Curves of Least Total Curvature, Sonderforschungsbereich, 382, Preprint Nr. 13, Tübingen 1995.

[9] A. Polden, Curves and Surfaces of Least Total Curvature and FourthOrder Flows, Dissertation, Universität Tübingen, 1996.

[10] L. Simon, Existence of Surfaces Minimizing the Willmore Functional, Commun. Analysis Geom., 1(2) (1993), 281-326.

[11] G. Simonett, The Willmore Flow near Spheres, preprint, 1999.

[12] M. Struwe, On the Evolution of Harmonic Maps of Riemannian Surfaces, Comment. Math. Helv., 60(2) (1985), 558-581.

[13] T. Willmore, Riemannian Geometry, Oxford University Press, New York, 1993.

[14] T. Willmore, Note on Embedded Surfaces, An. Stiint. Univ., "Al. I. Cusa" Iasi Sect. Ia, 11B (1965), 493-496.

Mathematisches Institut DeR

Albert-Ludwigs-Universität Freiburg

Eckerstrasse 1, D-79104 Freiburg, Germany

E-mail address: kuwert@mathematik.uni-freiburg.de

Mathematisches Institut DER

RHEINISCHEN FRIEDRICH-WILHELMS-UNIVERSITÄT BONN

BERINGSTRASSE 6

D-53115 BONN

GERMANY

E-mail address: schaetz@math.uni-bonn.de

Received February 4, 2000. 